\title{
Graph Theory Solution Method to Solve the Complex Assembly Dimension Chain
}

\author{
Ya Zhang ${ }^{1, a^{*}}$ and Lin Zhang ${ }^{2, b}$ \\ ${ }^{1}$ Zhejiang University of Science and technology, Hangzhou, 310027, China \\ ${ }^{2}$ Yangzhou Polytechnic College, Yangzhou, 225127, China \\ yazhang1982@126.com, b330213049@qq.com
}

Keywords: Dimension chain; Transferring coefficient; Matrix; Graph theory model

\begin{abstract}
Dimension chain analysis very important to assure the assembly quality of the engineering machines. If the engineering machine component has a lot of parts, the representation and solution of the assembly dimension chain is too difficult by general dimension chain analysis method. Based on the graph theory model, a method is proposed to solve the dimension chains of the complex assembly of engineering machines components. At last, an assembly practice of a roller holler is analyzed to validate the method.
\end{abstract}

\section{Introduction}

Graph theory is a new branch of mathematics. It has been widely used in physics, chemistry, operations research, computer science, information, network theory and economic management and many other fields currently ${ }^{[1]}$.In the field of mechanical assembly, graph theory is also attracting more and more people's attention $^{[2]}$.

Generally, we can use the principle of the dimension chains to solve the assembly dimensions. Dimensional chain analysis is an important method to ensure the quality of the machine assembly ${ }^{[3]}$. However, if there are a lot of parts in the assembly of mechanical components, the representation and solution of the dimension chains will be very difficult. According to the graph theory representation model of assembly dimension, this paper proposed a matrix solution method of the assembly dimension chain.

This method can represent all the dimensional relationships in a matrix form and solve all the closed-loop dimensions in one calculation. It provides an effective way for solving the complex dimension chain. In the following, we take the assembly of the roller components for an example to illustrate that the assembly dimension chain solution based on graph theory. 


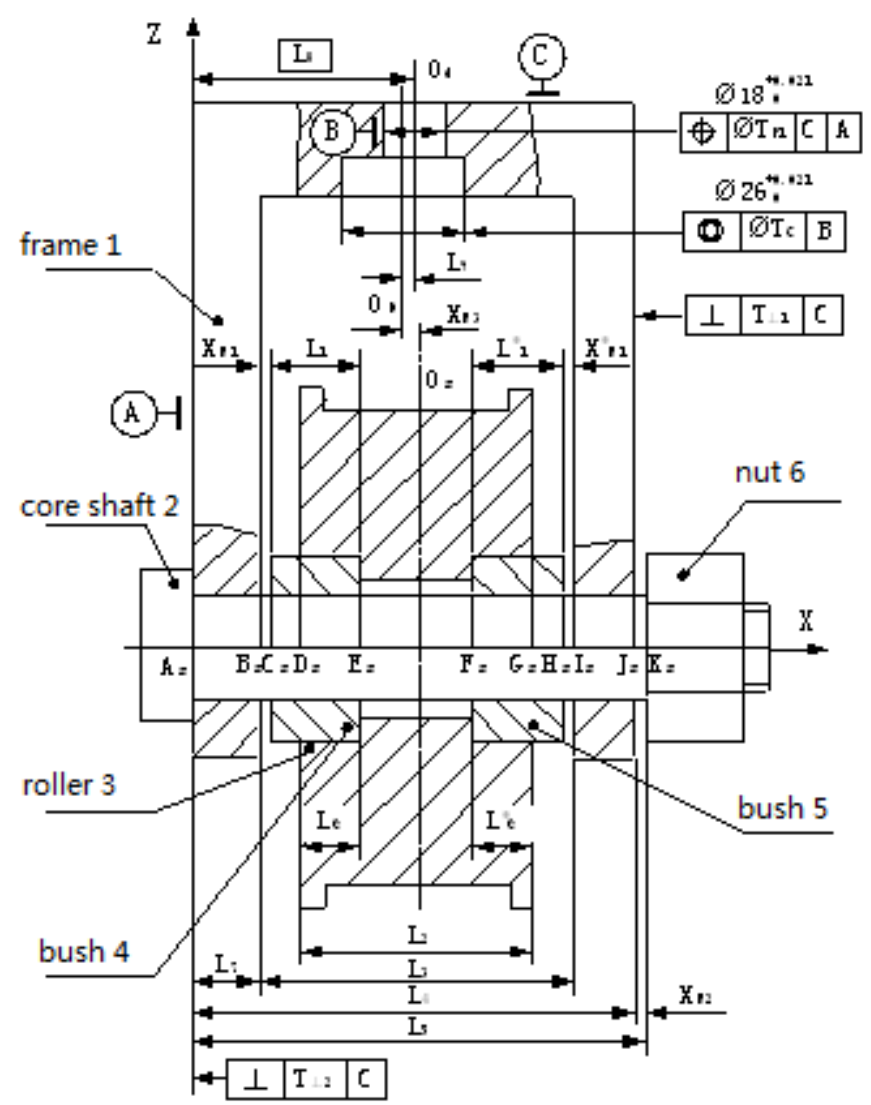

Figure 1 Roller assembly

\section{Graph Theory Model of Assembly Dimension Chain}

As shown in Figure. $1^{[4]}$, the wheel component has six parts. The design dimensions of the components are:

$\mathrm{L}_{1}=15 \mathrm{~mm}, \mathrm{~L}_{2}=38 \mathrm{~mm}, \mathrm{~L}_{3}=50 \mathrm{~mm}, \mathrm{~L}_{4}=80 \mathrm{~mm}, \mathrm{~L}_{5}=80.2 \mathrm{~mm}, \mathrm{~L}_{6}=10 \mathrm{~mm}, \mathrm{~L}_{7}=15 \mathrm{~mm}, \mathrm{~L}_{8}=40 \mathrm{~mm}$ and $\mathrm{L}_{9}=0$.

Based on the design dimensions of the parts, it is required to solve three assembly dimensions, which are the dimension of the closed loop:

The clearance $\mathrm{XN}_{1}$ between the roller 3 and the frame 1;

The clearance $\mathrm{XN}_{2}$ between the frame 1 and the nut 6 ;

The distance $\mathrm{XN}_{3}$ between the axis of the big hole $\Phi 26_{0}^{+0.021}$ of the rack 1 and the middle plane of the two end faces in the roller 3, which are perpendicular to the axis. The graph theory model of assembly dimension chain was established.

Figure. 1 can be defined as a binary group $G=\langle V(G), E(G)\rangle$

(1) $V(G)$ is a non-empty set, and $V(G)=\left\{v_{1}, v_{2}, \ldots, v_{n}\right\}$. The element $v_{i}(i=1,2, \ldots, n)$ represents the node of the graph $G$. This figure only contains the nodes that are related to the assembly dimensions and design dimensions. $V(G)$ is called the set of nodes of graph $G$, which is abbreviated as $V$.

$E(G)$ is an ordered set of nodes in $V, E(G)=\left\{e_{1}, e_{2}, \ldots, e_{m}\right\}=\left\{v_{i} v_{j}, i<j, i, j=1,2, \ldots, n\right\}$. Edge $e_{k}$ is associated with ordered pair $\left\langle v_{i} v_{j}\right\rangle . e_{k}$ is a directed edge, where $v_{i}$ is the starting node of $e_{k}$ and $v_{j}$ is the ending node of $e_{k}$. In order to determine the transfer coefficient of the composition of the ring, we designated the direction of the component ring (design size) is from the left node points to the right node points. It is defined $i<j$. Figure. 2 shows the diagram theory model of roller assembly, thick solid line in the figure represents that the assembly size is closed ring. 


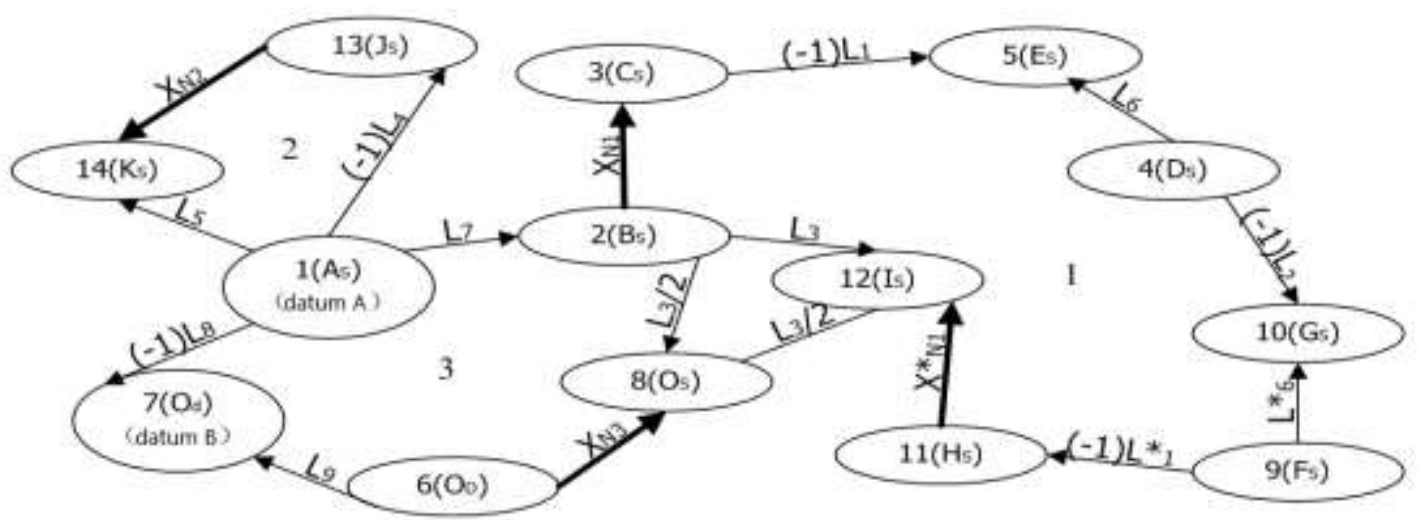

Figure.2 Assembly size of the graph theory model

\section{Solution of the Assembly Size Chain}

The steps to set up assembly dimension chain matrix are as follows:

First of all, using the node information in the graph theory model, find the transfer coefficient of each component ring. Secondly, according to the transfer coefficient and the edge of the weight information, establish the assembly size matrix. At last, solve the assembly size.

Determine the increasing and decreasing ring, and determine the composition of the ring transfer coefficient according to the node information in the figure. The transfer coefficient is the coefficient that indicates the influence of each component ring on the closed loop. The relationship between the closed loop and each component ring in the dimension chain can be expressed as: $L_{0}=f\left(L_{1}, L_{2}, \ldots, L_{m}\right)$. Let the transfer coefficient of the $i_{\text {th }}$ component ring be $\xi_{i}$, then $\xi_{i}=\partial f / \partial L_{i}$. For the increasing ring, $\partial f / \partial L_{i}$ and $\xi_{i}$ are positive; for reduction ring, $\xi_{i}$ is negative ${ }^{[1]}$. In the linear dimension chain, the transfer coefficient of the increasing ring is $\xi_{i j}=+1$, and the transfer coefficient of the reduced ring $\xi_{i j}=-1$.

The basic dimension of the closed ring is equal to the sum of the basic dimensions of the rings plus the sum of the basic dimensions of the rings:

$$
A_{0}=\sum_{i=1}^{m} \vec{A}_{i}-\sum_{i=m+1}^{n-1} \overleftarrow{A}_{i}
$$

For the assembly dimension chain with multiple closed loops (assembly dimensions), we can build the matrix as follows:

If an assembly dimensional graph theory model contains $i$ rings contains closed loops (assembly dimensions), the design dimensions are $L_{1} \sim L_{j}$ and the assembly dimensions are $X_{N 1} \sim X_{N i}$, then:

$$
\left[X_{N i}\right]^{T}=\left[\xi_{i j} k_{i j}\right]\left[L_{j}\right]^{T}
$$

In this example, $i=1,2,3 ; j=1,2, \ldots 9$.

So the dimension chain coefficient matrix can be represented as:

$$
\begin{aligned}
& \begin{array}{llllllllll}
\mathrm{L}_{1} & \mathrm{~L}_{2} & \mathrm{~L}_{3} & \mathrm{~L}_{4} & \mathrm{~L}_{5} & \mathrm{~L}_{6} & \mathrm{~L}_{7} & \mathrm{~L}_{8} & \mathrm{~L}_{9}
\end{array} \\
& 2\left[\begin{array}{ccccccccc}
-1 & -1 / 2 & 1 / 2 & 0 & 0 & 1 & 0 & 0 & 0 \\
0 & 0 & 0 & -1 & 1 & 0 & 0 & 0 & 0 \\
0 & 0 & 1 / 2 & 0 & 0 & 0 & 1 & -1 & 1
\end{array}\right]
\end{aligned}
$$

Therefore, the assembly dimension chain matrix equation can be obtained:

$$
\left[\begin{array}{lll}
X_{N 1} & X_{N 2} & X_{N 3}
\end{array}\right]^{T}
$$




$$
\begin{aligned}
& \quad\left[\begin{array}{ccccccccc}
-1 & -1 / 2 & 1 / 2 & 0 & 0 & 1 & 0 & 0 & 0 \\
0 & 0 & 0 & -1 & 1 & 0 & 0 & 0 & 0 \\
0 & 0 & 1 / 2 & 0 & 0 & 0 & 1 & -1 & 1
\end{array}\right] \quad\left[\begin{array}{llllllllll}
L_{1} & L_{2} & L_{3} & L_{4} & L_{5} & L_{6} & L_{7} & L_{8} & L_{9}
\end{array}\right]^{T}= \\
& \left.\quad=\quad \begin{array}{l}
-\mathrm{L}_{1}-\mathrm{L}_{2} / 2+\mathrm{L}_{3} / 2+\mathrm{L}_{6} \\
-\mathrm{L}_{4}+\mathrm{L}_{5} \\
\mathrm{~L}_{3} / 2+\mathrm{L}_{7}-\mathrm{L}_{8}+\mathrm{L}_{9}
\end{array}\right]
\end{aligned}
$$

In the software "Mathematica", for the dimension chain coefficient matrix, generate a blank matrix $i \times j$, and then input the values to obtain the size chain coefficient matrix. Assembly dimension chain closed loop column vector can be obtained:

$$
\left[\begin{array}{lll}
X_{N 1} & X_{N 2} & X_{N 3}
\end{array}\right]^{T}=\left[\begin{array}{lll}
1 & 0.2 & 0
\end{array}\right]^{T}(4)
$$

Therefore, the assembly dimension of this roller assembly is:

The value of the clearance $\mathrm{XN}_{1}$ between the roller 3 and the frame 1 is 1 ;

The value of the clearance $\mathrm{XN}_{2}$ between the frame 1 and the nut 6 is 0.2 ;

The value of the distance $\mathrm{XN}_{3}$ between the axis of the big hole $\Phi 26_{0}^{+0.021}$ of the rack 1 and the middle plane of the two end faces in the roller 3 is 0.

\section{Conclusion}

In the assembly process of machine, if the relationship between the dimensions is complex, the representation and solution of the dimension chain will be very difficult.

Sometimes, it is even impossible to solve assembly dimensions using the usual dimensional chain approach. According to the graph theory model of assembly dimension chain, this paper establishes the matrix of assembly dimension chain firstly. Then, a new method of judging the transfer coefficient of dimension chain in graph theory model is proposed. By using this method, assembly dimension chains can be easily solved. Through the assembly example of roller components, the effectiveness of the proposed method is verified.

\section{Acknowledgment}

This work was financially supported by Startup Foundation of Zhejiang University of Science and Technology (F701102H02).

\section{Reference}

[1] Searching and Calculating the Process Dimensional Chain Using Graphic Theory, Yin Chenglong, Journa 1 of Anh ui Ag ricultural Univ ersity, 1998, 25 (2) : 203-205

[2] Algorithm for Assembly Sequences Planning Using Graph Theory and Heuristic Search, JI Zhongqi, TONG Ruofeng, LIN Lanfen, CAI Ming, DONG Jinxiang, Computer Engineering, 2003/13: $115-117$

[3] Li Zhu, Xu Zhengao, Exchangeability \& Measurement Technology Jiang Xiangqian,Beijing: higher education press, 2004.

[4] A New Model for Concurrent Tolerancing in CAD System, Huang Meifa Zhong Yanru, 2004,15(18): $1623-1626$ 